\title{
Un modelo de aprendizaje multiprofesional en ciencias de la salud: innovación docente como respuesta a las necesidades emergentes de nuestra sociedad
}

\author{
Joaquim Colla, Dolors Bernabeu b, Cristina Cervantes c, Maria Nolla d, Josep M. Muniesac, \\ Jordi Tor ${ }^{a}$, Florentina Zapicob ${ }^{b}$, Natalia Pueyo ${ }^{d}$, Josep Nebot Cegarra ${ }^{a}$
}

\section{Introducción}

La Facultad de Medicina de la Universitat Autònoma de Barcelona (UAB) ha impulsado un nuevo modelo de enseñanza de las ciencias de la salud, un modelo integrador que aglutina los estudios de medicina, enfermería y fisioterapia en un mismo contexto educativo.

Desde el año 1969, la Facultad de Medicina imparte docencia en los estudios de medicina. Casi 40 años después de acoger la primera promoción de estudiantes de medicina, y coincidiendo con los cambios docentes que implica la Declaración de Bolonia y el nuevo Espacio Europeo de Educación Superior (EEES), la Facultad de Medicina ha optado por un proyecto pionero que tiene el objetivo de estimular los valores de solidaridad, tolerancia y el espíritu de servicio entre los futuros profesionales de las ciencias de la salud. Para conseguirlo, se han elaborado unos planes de estudio basados en la enseñanza y en la programación docente multiprofesional.

\section{Aprendizaje multiprofesional: hacia un nuevo modelo docente}

La Facultad de Medicina de la UAB dispone de una amplia red de infraestructuras docentes y asistenciales (Tabla I; Figura) que permite plantearse y cuestionar los modelos tradicionales de las facultades de medicina. Históricamente, los estudios de medicina se han basado en currículos de enseñanza uniprofesional, en los que los estudiantes de medicina alcanzan las competencias propias de su profesión, pero no comparten ninguna actividad docente, ni tampoco el mismo contexto físico, con los estudiantes de los otros ámbitos de las ciencias de la salud (enfermería, fisioterapia, terapia ocupacional) [1].

El aprendizaje uniprofesional es importante para desarrollar conocimientos, habilidades y actitudes propias de la profesión. Sin embargo, las rutinas sanitarias se basan en el trabajo en equipos interdisciplinarios, en la colaboración profesional y en la toma de decisiones en grupo, por lo que una interacción entre los futuros profesionales es crucial para garantizar un servicio asistencial de calidad y enfocado a la atención social [2].

Las razones principales para fomentar la enseñanza integradora entre estudiantes de diferentes perfiles profesionales son [3]:

- Modificar actitudes negativas y pensamientos estereotipados.

- Detectar deficiencias en la confianza y en la comunicación entre profesionales.

- Reforzar las competencias de colaboración y trabajo en equipo.

- Mejorar la calidad de los servicios asistenciales.

- Mejorar la satisfacción en el trabajo.

- Crear una fuerza laboral más flexible.

- Integrar las especializaciones y desarrollar una atención al paciente integral.

En este contexto, la Facultad de Medicina de la UAB ha optado por el aprendizaje compartido, en el que uno o más estudiantes de diferentes ámbitos de las ciencias de la salud aprenderán conjuntamente desarrollando competencias de trabajo en equipo.
Profesor del grado de Medicina.

b Profesor del grado de Enfermería. Profesor del grado de Fisioterapia.

d Unidad de Educación Médica. Universitat

Autònoma de Barcelona.

E-mail

joaquim.coll@uab.cat 


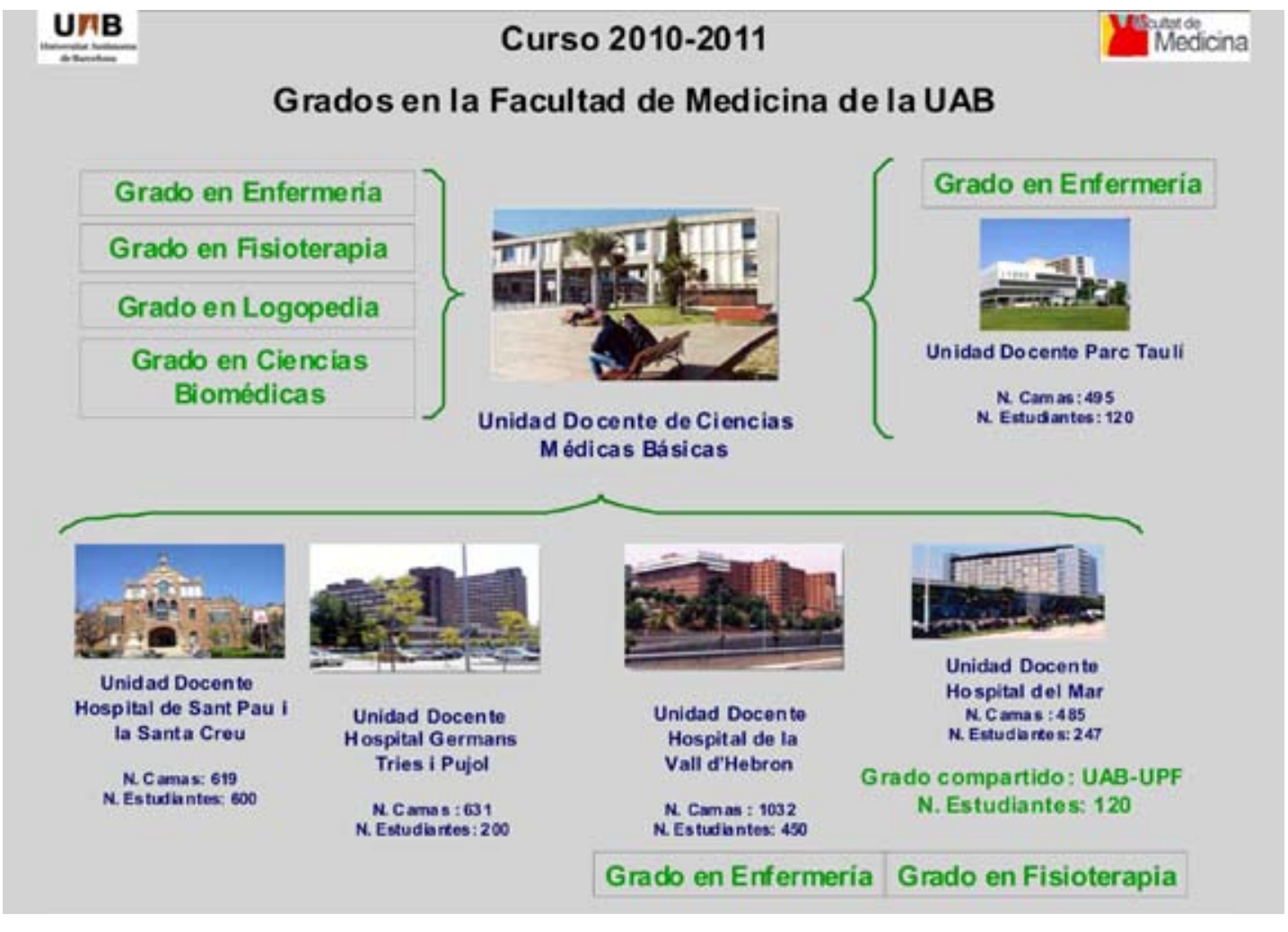

Figura. Grados en la Facultad de Medicina de la UAB.

Para alcanzar este objetivo, la facultad ha decidido acoger la docencia simultánea de los grados de Medicina, Enfermería y Fisioterapia. Reunir los estudiantes de estas ramas de las ciencias de la salud ha de permitir diseñar una docencia multiprofesional que proporcione a los estudiantes de las tres titulaciones las herramientas para adquirir habilidades, actitudes o conocimientos, compartiendo un mismo espacio físico y utilizando conjuntamente los mismos materiales de aprendizaje, a la vez que en determinadas materias podrán compartirse profesores con diversas formaciones profesionales.

Los estudiantes compartirán las instalaciones de la Unidad Docente de Ciencias Médicas Básicas, situada en el campus de la UAB, durante los primeros cursos de formación básica en el ámbito de las ciencias de la salud, para que alcancen los siguientes resultados de aprendizaje:

- Demostrar la comprensión de los roles, las expectativas y los límites de la salud de otras profesiones sanitarias.
- Identificar los principios clave que facilitan el trabajo interprofesional eficaz.

- Explicar cómo las mejoras en el trabajo interprofesional pueden ser importantes para el paciente.

- Discutir y reflexionar sobre las oportunidades y limitaciones provocadas por las percepciones que los profesionales de la salud tienen unos de otros.

- Proporcionar y evaluar modelos de colaboración entre la salud y diversas profesiones de atención social.

- Analizar y criticar los modelos de aprendizaje interprofesional y de trabajo interprofesional.

Con esta estrategia, la Facultad de Medicina pretende convertirse en un centro docente de innovación curricular que marque una nueva forma de comprender la formación de los futuros profesionales de las ciencias de la salud.

La Facultad de Medicina no sólo acoge las tres titulaciones de Medicina, Enfermería y Fisioterapia, sino que también participa en la formación 
de los graduados de Medicina (grado compartido con la Universitat Pompeu Fabra), de los graduados en Ciencias Biomédicas (docencia compartida con la Facultad de Biociencias) y de los graduados de Logopedia (docencia compartida con la Facultad de Psicología).

El modelo docente de la Facultad de Medicina de la UAB se acerca a modelos de aprendizaje multiprofesionales que ya se han implementado desde hace unos años en otros centros, como la Universidad de Linköping (Suecia) -que ofrece un curso de diez semanas, denominado 'Man in Society', que está abierto a la participación de estudiantes de medicina, enfermería, fisioterapia, trabajo social y terapia ocupacional-, y la Universidad de Bristol (Reino Unido), la Universidad de British Columbia (Canadá), la Universidad de Newcastle (Australia), la Universidad de Nevada (Estados Unidos), y la Universidad de San Bart's (Reino Unido), que ofrecen cursos compartidos por estudiantes de medicina, enfermería, fisioterapia y trabajo social [2].

\section{Estrategia de implementación}

La estrategia que plantea la Facultad de Medicina abarca diferentes fases de implementación del aprendizaje multiprofesional.

Durante la primera fase, el objetivo de la facultad ha sido conseguir que se impartan los grados de Medicina, Enfermería y Fisioterapia en la Unidad Docente de Ciencias Médicas Básicas. Durante dos años se ha estado trabajando en diferentes comisiones, un proceso que ha culminado con la aprobación, por parte de la Agencia Nacional de Evaluación de la Calidad y Acreditación (ANECA), de los planes de estudio presentados por la Facultad de Medicina: grados de Medicina, Enfermería y Fisioterapia.

Desde el curso 2009-2010, la Facultad de Medicina imparte el grado de Enfermería y la licenciatura de Medicina (a partir del curso 2010-2011 será el grado de Medicina) en la Unidad Docente de Ciencias Médicas Básicas.

Este primer año de integración del grado de Enfermería en la facultad ha provocado un cambio en la forma de comprender la docencia, ya que los profesores propios de las ciencias médicas básicas han estado trabajando conjuntamente con los profesores vinculados al Departamento de
Tabla I. Recursos asistenciales (camas) al servicio de la docencia en la Facultad de Medicina de la UAB.

\begin{tabular}{|c|c|}
\hline Unidades docentes & 3.226 \\
\hline Hospitales concertados & 1.808 \\
\hline Institutos universitarios & 350 \\
\hline Total & 5.384 \\
\hline Camas de agudos & 3.769 \\
\hline Camas sociosanitarias (30\%) & 1.615 \\
\hline Centros de Atención Primaria & 64 \\
\hline
\end{tabular}

Enfermería (de nueva creación) en el diseño de las asignaturas.

La enseñanza multiprofesional ha sido el mayor reto en esta primera fase, en la que se ha puesto en marcha un diálogo sobre las metodologías docentes, las competencias y los contenidos de las asignaturas, generando nuevos impulsos y enriqueciendo las propuestas docentes tradicionales con módulos compartidos entre estudiantes de medicina y de enfermería y otras actividades optativas de enseñanza multiprofesional.

Estas primeras aproximaciones a un aprendizaje integrado entre medicina y enfermería han constituido una prueba piloto que se continuará desarrollando con la integración de los estudios del grado de Fisioterapia en la facultad, que coincide también con el primer curso del grado de Medicina que empezará a impartirse a partir del curso 2010-2011. Un total de 540 estudiantes de nuevo ingreso se incorporarán a la facultad durante este curso. Las infraestructuras de la Facultad de Medicina permiten una gestión flexible de los estudios y la adaptación a las futuras demandas sociales.

Teniendo en cuenta que el diseño del currículo según las directrices del EEES ha permitido configurar los primeros cursos de los grados con asignaturas de formación básica en ciencias de la salud, se fomenta la programación de espacios de integración y de aprendizaje multiprofesional. 
En la tabla II se muestran las asignaturas de formación básica de los tres grados. Se señalan en cursiva las asignaturas susceptibles de permitir una docencia compartida, total o parcialmente, entre los diferentes perfiles de estudiantes de la facultad, en base a la disponibilidad de profesorado, la respuesta de los propios estudiantes, la evaluación periódica y los resultados obtenidos en el proceso de aprendizaje. Dada la gran innovación docente que esto ha de suponer, se opta por un desarrollo progresivo del programa de innovación.

Actualmente, en algunas asignaturas ya se están diseñando módulos de aprendizaje multiprofesional donde los profesores podrán trabajar con equipos mixtos de diferentes titulaciones.

Otras formas de enseñanza multiprofesional que se pretende llevar a cabo, a corto plazo, son:

- Seminarios compartidos entre las tres titulaciones.

- Prácticas compartidas en el aula de habilidades.

- Actividades optativas y complementarias a las asignaturas.

En general, la Facultad de Medicina de la UAB continuará trabajando para implementar, progresivamente, el aprendizaje multiprofesional. Las diferentes fases de este proceso, que abarcará los próximos 5-6 años, se relacionan en la tabla III.

\section{Modelos docentes de la Facultad de Medicina de la UAB}

Además del aprendizaje multiprofesional, la Facultad de Medicina también opta por otros modelos de innovación curricular.

\section{Grado de Medicina}

La formación básica se realiza entre el primer y el segundo curso. La formación nuclear clínica de la titulación se distribuye entre tercero, cuarto y quinto, y el sexto año se dedica, básicamente, al rotatorio de prácticas clínicas y al trabajo de fin de grado. Las asignaturas optativas se pueden cursar entre segundo y sexto curso.

El grado de Medicina se estructura siguiendo cinco principios básicos:

- Concentrar la formación básica en los primeros dos cursos.
- Liberar el último curso de asignaturas obligatorias para facilitar al estudiante una inmersión completa en los servicios y centros asistenciales durante las prácticas clínicas tuteladas.

- Incorporar el aprendizaje integrado en medicina en el plan de estudios.

- Fomentar el contacto precoz de los estudiantes con el sistema sanitario, desde el primer curso.

- Estructurar la oferta de asignaturas optativas en menciones.

El estudiante podrá optar por una de las siguientes menciones:

- Mención en Clínica Médica.

- Mención en Cirugía Clínica.

- Mención en Laboratorio Clínico y Experimental.

- Mención en Salud Materno-Infantil.

- Mención en Salud Pública y Servicios de Salud.

- Mención en Salud Mental.

La apuesta por las menciones en el grado de Medicina tiene en cuenta el discurso social existente en este momento sobre la necesidad de troncalidad en la formación especializada. El plan de estudios ha optado por un modelo innovador en el cual las asignaturas optativas clásicas permiten al alumno iniciarse en un cierto componente de troncalidad para su futura especialización.

\section{Grado de Enfermería}

La formación básica se realiza mayoritariamente en el primer curso. La formación nuclear de la titulación se distribuye básicamente entre segundo y tercero, y en el cuarto año se desarrollan las materias optativas, las prácticas clínicas y el trabajo de fin de grado.

El modelo docente del grado de Enfermería se basa en los siguientes principios:

- Basar la docencia en la metodología de aprendizaje basado en problemas.

- Facilitar al estudiante la integración en los servicios y centros asistenciales durante las prácticas clínicas.

- Estructurar la oferta de asignaturas optativas en menciones.

El estudiante podrá optar por una de las siguientes menciones: 
Tabla II. Asignaturas de formación básica de los grados de Medicina, Enfermería y Fisioterapia (en cursiva, asignaturas susceptibles de ser parcial o totalmente compartidas).

\begin{tabular}{|c|c|c|c|c|c|}
\hline Curso & Sem. & Asignatura & Carácter & ECTS & Materia \\
\hline \multirow{6}{*}{1} & \multirow[t]{2}{*}{1} & Introducción a las ciencias de la salud & $\mathrm{FB}$ & 6 & $\begin{array}{l}\text { Propedéutica de las } \\
\text { ciencias de la salud }\end{array}$ \\
\hline & & Biología celular & $\mathrm{FB}$ & 6 & Biología \\
\hline & \multirow{4}{*}{ Anual } & Anatomía humanal & FB & 9 & Anatomía humana \\
\hline & & Biofísica & $\mathrm{FB}$ & 7 & Física \\
\hline & & Bioestadística & $\mathrm{FB}$ & 6 & Estadística \\
\hline & & Bioquímica y biología molecular humana & $F B$ & 12 & Bioquímica \\
\hline \multirow{6}{*}{2} & \multirow[t]{2}{*}{1} & Psicología médica & FB & 6 & Psicología \\
\hline & & Fisiología médica l & FB & 8 & Fisiología \\
\hline & 2 & Fisiología médica ll & $\mathrm{FB}$ & 8 & Fisiología \\
\hline & \multirow{2}{*}{ Anual } & Estructura microscópica de aparatos y sistemas & $\mathrm{FB}$ & 6 & Histología \\
\hline & & Anatomía humana II & $\mathrm{FB}$ & 9 & Anatomía humana \\
\hline & & Total formación básica & & 83 & \\
\hline \multicolumn{6}{|c|}{ Enfermería } \\
\hline \multirow{8}{*}{1} & \multirow{5}{*}{1} & Estructura del cuerpo humano & $\mathrm{FB}$ & 6 & Anatomía humana \\
\hline & & Ciencias psicosociales & $\mathrm{FB}$ & 6 & Psicología \\
\hline & & Función del cuerpo humano I & $\mathrm{FB}$ & 6 & Fisiología \\
\hline & & Metodología científica y bioestadística & $\mathrm{FB}$ & 6 & Estadística \\
\hline & & Cultura, sociedad y salud & FB & 6 & Sociología \\
\hline & \multirow{3}{*}{2} & Comunicación y TIC & $\mathrm{FB}$ & 6 & Comunicación \\
\hline & & Función del cuerpo humano II & $\mathrm{FB}$ & 6 & Fisiología \\
\hline & & Nutrición & FB & 6 & Nutrición \\
\hline \multirow{3}{*}{2} & 1 & Comunicación terapéutica & FB & 6 & Psicología \\
\hline & 2 & Farmacología & FB & 6 & Farmacología \\
\hline & & Total formación básica & & 66 & \\
\hline \multicolumn{6}{|c|}{ Fisioterapia } \\
\hline \multirow{7}{*}{1} & \multirow{5}{*}{1} & Anatomía humanal & $\mathrm{FB}$ & 6 & Anatomía humana \\
\hline & & Bases biológicas del cuerpo humano & FB & 9 & Fisiología \\
\hline & & Biofísica y biomecánica & $\mathrm{FB}$ & 6 & Biofísica \\
\hline & & Metodología científica y bioestadística & $\mathrm{FB}$ & 6 & Estadística \\
\hline & & Función del cuerpo humano & FB & 9 & Fisiología \\
\hline & \multirow[t]{2}{*}{2} & Anatomía humana ll & $\mathrm{FB}$ & 6 & Anatomía humana \\
\hline & & Psicología humana & $F B$ & 6 & Psicología \\
\hline \multirow{3}{*}{2} & 1 & Conceptos clínicos patológicos. Técnicas de diagnóstico & $\mathrm{FB}$ & 6 & Patología humana \\
\hline & 2 & Patología medicoquirúrgica & $\mathrm{FB}$ & 6 & Patología humana \\
\hline & & Total formación básica & & 60 & \\
\hline
\end{tabular}


Tabla III. Fases en el aprendizaje multiprofesional en la Facultad de Medicina de la UAB.

\begin{tabular}{ll}
\hline Fase & Objetivo \\
\hline 1. Coexistencia multiprofesional & $\begin{array}{l}\text { Compartir el material docente entre las asignaturas de las titulaciones } \\
\text { Elaborar material docente que pueda servir para los tres grados }\end{array}$ \\
\hline 2. Aprendizaje multiprofesional por módulos & $\begin{array}{l}\text { Compartir módulos de asignaturas } \\
\text { Compartir las prácticas en el aula de habilidades }\end{array}$ \\
\hline 3. Aprendizaje multiprofesional por asignaturas & $\begin{array}{l}\text { Compartir asignaturas (p. ej., metodología científica y bioestadística, } \\
\text { psicología humana...) }\end{array}$ \\
\hline 4. Aprendizaje multiprofesional integral & $\begin{array}{l}\text { Compartir todas las asignaturas de formación básica entre las tres } \\
\text { titulaciones }\end{array}$ \\
\hline
\end{tabular}

- Mención en Infancia y Salud.

- Mención en Vejez y Salud.

- Mención en Salud Mental a lo largo de la vida.

- Mención en Salud y Comunidad.

\section{Grado de Fisioterapia}

La formación básica se realiza mayoritariamente en el primer curso. La formación nuclear de la titulación se distribuye básicamente entre segundo y tercero, y en el cuarto año se desarrollan las materias optativas, las prácticas externas y el trabajo de fin de grado.

El modelo docente del grado de Fisioterapia se basa en los siguientes principios:

- Ofrecer una formación en fisioterapia en el ámbito público y adaptada a las necesidades emergentes de la sociedad.

- Facilitar al estudiante la integración en los servicios y centros asistenciales durante las prácticas clínicas.

- Estructurar la oferta de asignaturas optativas en menciones.

El estudiante podrá optar por una de las siguientes menciones:

- Mención en Fisioterapia del Aparato Locomotor.

- Mención en Fisioterapia Neurológica.

- Mención en Fisioterapia Cardiaca y Respiratoria.

\section{Aportaciones de innovación docente de la Facultad de Medicina de la UAB}

En 1988, la Organización Mundial de la Salud [4] ya destacó que si los profesionales de la salud aprenden a colaborar juntos desde la etapa de estudiantes, también tendrán más facilidad de trabajar conjuntamente en equipos clínicos o laborales.

La integración de los estudios de medicina, enfermería y fisioterapia en un mismo espacio docente acerca la visión de los estudiantes a la realidad asistencial multiprofesional, ya que se acabarán incorporando como profesionales dentro del ámbito de la salud, demostrando unos valores basados en la empatía, el respeto y la comprensión del paciente.

Ante este gran reto docente, las aportaciones de innovación docente básicas que se ha propuesto fomentar en la facultad son:

- La docencia basada en un modelo multiprofesional donde se integren los estudiantes de las tres titulaciones (Medicina, Enfermería y Fisioterapia), para compartir y construir su aprendizaje de forma activa, aprendiendo unos junto a otros.

- El contacto precoz con el sistema sanitario, facilitando al estudiante que se familiarice con el primer nivel del sistema sanitario, los Centros de atención primaria y centros sociosanitarios (dependencia, geriatría, salud mental, entre otros). 
- La creación de asignaturas integradas, con el objetivo de ofrecer una visión holística de las ciencias de la salud al estudiante.

- La metodología de aprendizaje basado en problemas, que otorga un rol activo al estudiante.

- La oferta de asignaturas optativas en menciones, ofreciendo al estudiante la posibilidad de profundizar en los ámbitos de su interés.

Además de estas estrategias curriculares, un punto clave en la gestión de los estudios es la gran red de infraestructuras de las que dispone la Facultad de Medicina de la UAB, y que permite adaptarse fácilmente a las demandas sociales en el ámbito de las ciencias de la salud. Así, el aumento (o reducción) de la oferta de plazas de las tres titulaciones, según las demandas actuales del sector, se puede llevar a cabo con mucha flexibilidad y un gasto público mínimo.

En conclusión, el modelo de innovación docente que se pretende aplicar permite afrontar un reto social y económico de gran importancia en estos momentos. Se trata de un sistema docente innovador que se adapta a las directrices del EEES $\mathrm{y}$ a las necesidades sociales de profesionales de nuestro país. Además, el modelo sigue las reco- mendaciones de la Organización Mundial de la Salud en cuanto a la enseñanza multiprofesional: que estudien juntos los que trabajarán juntos en un futuro.

\section{Bibliografía}

1. McKimm J, Brake DJ. Interprofessional education. London: London Deanery; 2010. URL: http://www.faculty. londondeanery.ac.uk/e-learning/interprofessional-education. [16.05.2010].

2. Freeth D. Understanding medical education guide: interprofessional education. Association for the Study of Medical Education (ASME). Edinburgh: ASME; 2007.

3. Barr H. Interprofessional education: today, yesterday and tomorrow. London: Learning and Teaching Support Network, Centre for Health Sciences and Practice; 2002. URL: http://meds.queensu.ca/quipped/assets/IPE Today, Yesterday \& Tmmw (Barr).pdf. [16.05.2010].

4. Grupo de Estudio de la Organización Mundial de la Salud. Aprender juntos a trabajar juntos por la salud: informe de un grupo de estudio de la OMS sobre educación multiprofesional del personal de salud, el criterio de equipo. Geneva: OMS; 1988. 